\title{
A LOW DISPERSION TURN FOR MINIATURIZED ELECTROPHORESIS
}

\author{
Joshua I. Molho, Amy E. Herr, Bruce P. Mosier, \\ Juan G. Santiago, and Thomas W. Kenny \\ Mechanical Engineering Department, Stanford University \\ Stanford, CA 94305-4021 \\ Reid A. Brennen and Gary B. Gordon \\ Agilent Laboratories, Agilent Technologies \\ Palo Alto, CA 94304-1392
}

\begin{abstract}
Many chip-based microcolumn separation systems require serpentine channels to obtain longer separation lengths within a compact area. However, analyte bands traveling through curved channels experience increased dispersion and thus, serpentine channels reduce the intended benefit of the increased channel length. We have used simulation tools to predict this increased band dispersion and then to design a corner geometry that causes less dispersion. Experiments using bleached- and cagcd fluorescence visualization were conducted to verify the proposed turn designs. We demonstrate experimentally that the new corner design greatly reduces dispersion of analyte bands. Quantitative and qualitative comparisons can be made between the simulations and experiments, and these results suggest that the simulations can be used effectively as design tools.
\end{abstract}

\section{INTRODUCTION}

Pioneered in the carly 1990's [1], on-chip capillary electrophoresis (CE) remains an important separation technique for chemical/biochemical microfluidic devices. This technique separates a mixture of unknown analytes based on their mobilities in an applied electric field. The basic scheme involves introducing a small volume of mixed, unknown analytes into a separation channel. An electric field is applied along the length of the separation channel and the various analytes move at different speeds based on their mobilites. After some time, the analytes separate into individual bands, as depicted schematically in Figure 1. The arrival of individual analyte bands can be detected downstream, often using a single point fluorescence or absorption sensor. The presence of a particular analyte in the unknown mixture can be determined by using markers injected with the sample or by first calibrating the system with a mixture of known analytes.

Several studies have demonstrated the potential benefits of miniaturizing capillary electrophoresis on microfabricated chips. Among these benefits are portability, reduced reagent use, and increased opportunities for parallelism [2]. However, since separation efficiency increases with the length of the separation channel, it is desirable to use longer microchannels within a small area, thus requiring turns in the microchannel. Recently, Culbertson et al. [3] have shown that such turns increase the dispersion of samples flowing in the microchannels and thus cancel the benefit of the additional length.

\section{THEORY}

Consider the electrophoretic separation of a fluorescentlytagged sample as depicted in Figure 1, where the initially mixed sample has separated into its two components some time after the application of the axial electric field. The two analyte bands are separated by a distance $\Delta L$ and the Gaussian bands have a standard deviation of $\sigma$. We can define a resolution as:

$$
\text { resolution }=\frac{\Delta L}{\sigma} \sim \frac{E \cdot \Delta \mu \cdot t}{\sqrt{D \cdot t}}
$$

where $\Delta \mu$ is the difference between the electrophoretic mobilities of the two analytes, $D$ is the diffusion coefficient and $t$ is the time of separation. In the common case where different analytes have different diffusion coefficients, the largest $\sigma$ can be used to define the resolution.

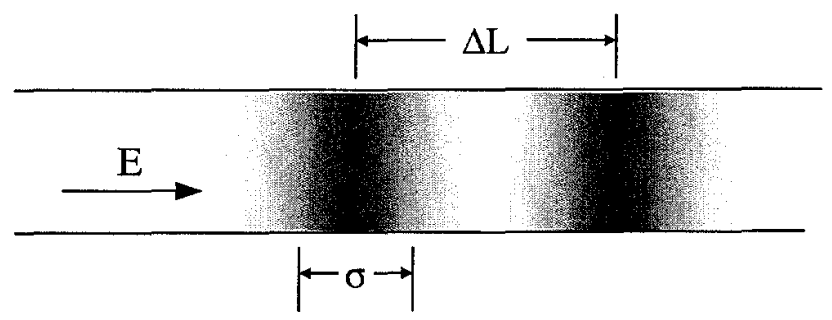

Figure 1. Schematic of an electrophoretic separation. Initially, the two bands shown above were mixed together. After the application of an electric field, the bands move from left to right and separate based on the difference in their electrophoretic mobilities.

The bands will travel a distance $L$ proportional to the separation time and therefore, we can also state that

$$
\frac{\Delta L}{\sigma} \sim E \cdot \Delta \mu \cdot \sqrt{L}
$$

Eq. 2 shows that the resolution will vary linearly with the field strength and mobility difference. The mobility difference is a property of the analytes and is not controlled. It is often advantageous to increase the field strength, but in some cases, further increases in the field strength will cause excessive Joule heating [4]. In such thermally limited cases, increasing the separation length will enhance the separation resolution. However, serpentine channels are required to increase separation length within a compact area.

Unfortunately, not all serpentine channels will increase separation resolution. Culbertson et al. [3] have presented a study of dispersion caused by constant radius turns where the radius of 
curvature is larger than the width of the channel. The general effect is illustrated in Figure 2.

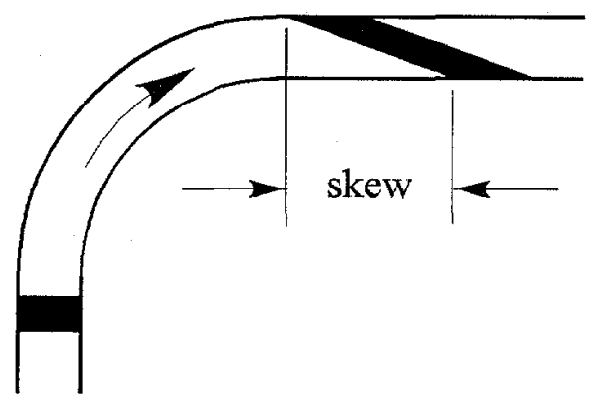

Figure 2. Effect of 90 degree, constant radius turn on an analyte band. The band is "skewed" as it travels through the turn with molecules near the inside of the turn racing ahead of the molecules near the outside of the turn. This skewing of the analyte band has been called the race-track effect.

The skew seen in Figure 2 is caused by the fact that the pathline along the inner portion of the turn is shorter than the pathline along the outer portion of the turn, and that the electric field is stronger along the inside of the turn. Using the approximate expression given by Culbertson et al. [3] the skew, as defined in Figure 2 and in the limit of no diffusion, is equal to

$$
\text { skew }=2 \theta w
$$

where $w$ is the width of the channel and $\theta$ is the angle completed by the turn (in radians). To address how diffusion will affect the amount of skew caused by a turn, we refer to the two-dimensional, non-dimensionalized advection-diffusion equation (Eq. 4) where $u^{\prime}, c^{\prime}, x^{\prime}$, and $y^{\prime}$ are the normalized velocity, concentration, direction along the channel and direction across the channel, respectively.

$$
\frac{\partial c^{\prime}}{\partial t^{\prime}}+\underbrace{u^{\prime} \frac{\partial c^{\prime}}{\partial x^{\prime}}}_{\text {advection }}=\frac{1}{P e_{w}}[\underbrace{\frac{w}{L\left(\frac{\partial^{2} c^{\prime}}{\partial x^{\prime 2}}\right)}+\underbrace{\frac{L}{w}\left(\frac{\partial^{2} c^{\prime}}{\partial y^{\prime 2}}\right)}_{\text {diffusion }}}_{\begin{array}{c}
\text { axial } \\
\text { diffusion }
\end{array}}]
$$

The Peclet number, $P e_{w}$ is a non-dimensional parameter that characterizes the relative importance of diffusion of analytes compared to advection of analytes; $\mathrm{Pe}=U w / D$ with $U$ being the average electrokinetic velocity around the turn, $w$ the width of the channel and $D$ the diffusivity of the analyte traveling through the channel. $L$ is a length scale in the axial direction along the channel. In this study, $L$ is the axial length of the turn, which is on the order of 10 times $w$.

When $P e_{w}$ is very small, the diffusion terms on the right side of Eq. 4 will dominate the evolution of the shape of the analyte band. In this paper, $\mathrm{L} / \mathrm{w}$ is on the order of 10 , so we see that when $P e_{w}$ is greater than 100 , the advection term dominates the shape of the analyte band. Designing the geometry of a compensating turn can be interpreted as an attempt to control the final shape of the analyte band through $u$ ' which appears in the advection term. Therefore, a compensating corner is most relevant and most effective when considering the case of large $P e_{w}$ (large molecules and/or large electrokinetic velocity). Note that when $P e_{w}$ is order 10 , the advection and the transverse diffusion terms are on the same order, and we cannot state, in general, that a corner designed for high $P e_{w}$ will be equally effective in this regime. In our approach we will design the corners to perform best at high $P e_{w}$, because as stated above, the modified $u$ ' will have the most effect in this regime. Furthermore, high $P e_{w}$ is representative of most on-chip separation systems. For example, a chip based separation system with $100 \mu \mathrm{m}$ wide channels, separation velocities on the order of $100 \mu \mathrm{m} / \mathrm{sec}$, and analytes with diffusivities on the order of $10^{-10} \mathrm{~m}^{2} / \mathrm{sec}$ (e.g. serum albumin) would have a $P e_{w}$ equal to 1000 . In the work presented in this paper, we have attempted to perform all the experiments and simulations in the limit high $P e_{w}$ on the order of 100 or higher.

The model for skew presented above does not directly account for the effect of electroosmosis in the turn. Most microchannel surfaces form a charged electric double layer when exposed to ionic solutions. Electroosmosis describes the bulk motion of fluid that results due to electrostatic body forces in the double layer when an axial electric field is applied [5]. In general, solving for the electroosmotic velocity requires solution of the electric field and charge density in the channel, followed by a solution to the Navier-Stokes equation.

Cummings et al. [6] suggest that in the case of uniform zeta potential, the electroosmotic velocity is everywhere parallel to the electric field. This result is valid when: the electric double-layers are thin compared to the channel width, no current passes through the walls of the channel, and the flow is parallel to the channel at any inlet or outlet to the region of interest. Utilizing this simplification, we propose to model the flow field by considering the electrophoretic component which, away from the electrodes, is similar to the electric field and the electroosmotic flow field. We demonstrate the validity of this approximation by using our flow measurement techniques.

\section{SIMULATION APPROACH}

Using the approximations described above, we have employed simulation tools (MEMCAD 4.6, Microcosm Technologies, Inc.) to design a low dispersion turn.

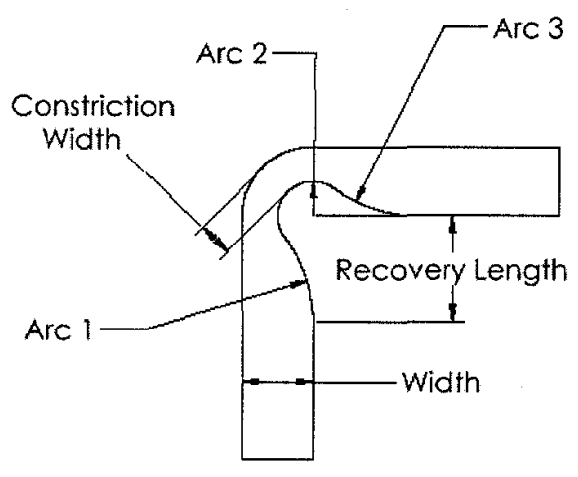

Figure 3. Parameterization of turn geometry. The corner is fully defined by the ratios of constriction to width and recovery length to width. Arcs 1,2 and 3 are all tangent to each other and Arcs 1 and 3 are tangent to the sides of the straight channels away from the corner. These final tangent conditions fully define the geometry once the geometric ratios have been defined. 
To compensate for the race-track effect, we propose to lengthen the pathline along the inner portion of the curve and to reduce transverse field gradients, using a modified turn as depicted in Figure 3. The corner design, as shown in Figure 3, is fully defined by specifying two ratios: the ratio of the width to recovery length and the ratio of the constriction width to the full channel width. This simple parameterization enables optimization of the corner design.

The simulated electric field strength in a compensating corner is compared to that of a constant radius corner in Figure 4. The compensating corner has a lengthened pathline along the inside of the turn and furthermore, a charged molecule that approaches the turn along the inside edge remains in a region of lower electric field strength (note the tilt of the contour lines) before reaching the higher field strength at the constriction. These two effects combine to help equalize the travel times of particles on the inside and outside edges of the corner, resulting in less skew.

Once the geometry is defined, the final shape of the analyte band is fully specified by the Peclet number. Recall that high $P e_{w}$ number corresponds to large molecules and/or high field strengths, and low $P e_{w}$ number corresponds to small molecules and/or low field strengths.
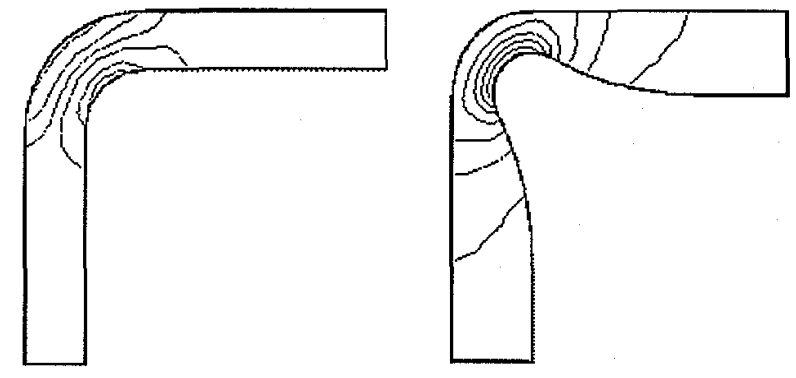

Figure 4. Comparison of electric field contours in a constant radius (left) and a compensating corner (right). Contours of constant electric field strength are shown for the two geometries.

To determine the final shape of an analyte band after it has passed through a corner design, the numerical solver performs the following steps. First, the electric field inside the channel is solved assuming electrically insulating sidewalls, and the inlet and outlet of the channel are held at constant potential. The straight sections of the channel before and after the turn must be long enough that imposing a uniform potential at the inlet and outlet will not perturb the solution (typically 3 to 4 channel widths). Next, the electrokinetic velocity in the channel can be determined by simulating only electrophoresis after appropriately modifying the mobility of the tracer dye to include the effects of electroosmosis. Finally, an initially Gaussian distribution of dye is numerically injected near the inlet of the corner and then tracked as it travels through the corner.

\section{EXPERIMENTAL SETUP}

To validate our design process and investigate the performance of our designs, channels were fabricated in polymethyl methacrylate (PMMA) using a 125-micron diameter end-mill. A PMMA coverslip was thermally bonded at $120^{\circ} \mathrm{C}$ onto the machined piece to complete the fluidic channels. Figure 5 shows the custom fixture that allows for pressure filling of liquids into the channels and that provides convenient wells for inserting electrodes.
The dispersion caused by the compensating corner was observed experimentally using bleached fluorescence [7] and caged fluorescence $[8,9]$ techniques as depicted in Figure 6. Both techniques provide an "optical injection" of a tracer. The caged fluorescence technique relies on caged dyes that are not fluorescent until uncaged with a UV pulse. The channels are filled with

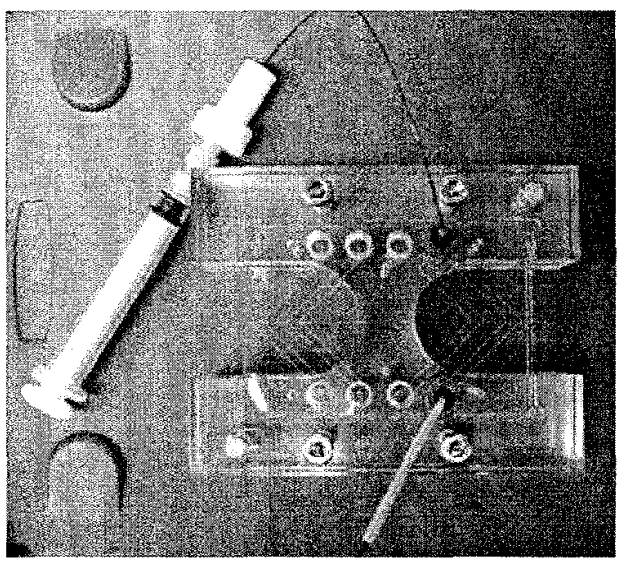

Figure 5. Custom fixture used during experimentation. This photograph shows the fixture that was used to position the microchannel chip and to provide seals for getting liquids into the chip. The eight channels can be seen as the faint traces on the $2 X$ 3 inch PMMA chip held in the fixture. The cutouts in the fixture provided clearance for the microscope objective used to image the corner designs.

a $400 \mu \mathrm{M}$ solution of caged fluorescein (Molecular Probes, Inc.) dissolved in de-ionized water. The uncaging is performed using a single pulse from a frequency tripled Nd:YAG laser $(355 \mathrm{~nm}, 500$ $\mu \mathrm{J}$ ) focused to an approximately $100 \mu \mathrm{m}$ by $2 \mathrm{~mm}$ area. For the bleaching technique, the channels are filled with a $10 \mu \mathrm{M}$ solution of a 2 MDalton dextran-fluorescein conjugate (Molecular Probes, Inc.) dissolved in de-ionized water. A 0.5 Watt argon ion continuous wave laser beam is focused through a $10 \mathrm{X}$ microscope objective $(\mathrm{NA}=0.3$ ) and translated across the channel to create a bleached timeline.

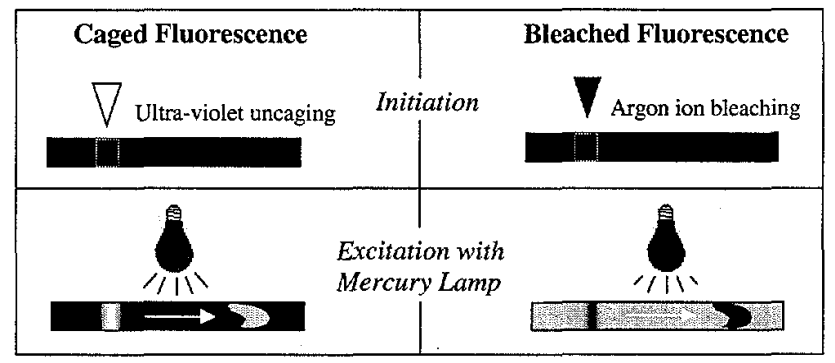

Figure 6. Schematic of caged (left) and bleached (right) fluorescence visualization techniques. The top row describes the "optical injection" stage of both techniques. The bottom row depicts the interrogation phase of the techniques when the marked region of the flow is tracked using epi-fluorescent microscopy.

After initiation, the marked region is excited with a Mercury lamp and images are collected at $10 \mathrm{X}$ magnification with an epifluorescent microscope and a $652 \times 488$, video rate, backilluminated CCD camera (PixelVision Inc.).

The bleaching method has the advantage of using any fluorescent dye, but it requires a relatively long time to bleach a region (order of tens to hundreds of milliseconds). The caged 
method requires custom dyes, but the UV pulse is short and therefore the uncaging can be performed while the electric field is applied.

\section{RESULTS AND DISCUSSION}

To evaluate the effectiveness of each design we use two quantities to describe the dispersion caused by the corner: the skew parameter and the full width at half maximum (FWHM) of the band. The FWHM of the band gives some indication of the dispersion caused by the corner by convolving a theoretical line detector with the band after it has traveled through the turn.

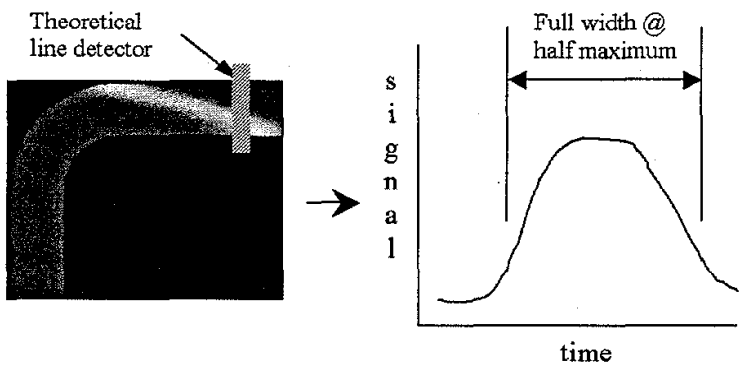

Figure 7. Determination of FWHM. The FWIIM calculation as shown here simulates convolving the perturbed analyte band shape with an ideal line detector.

The theoretical line detector can be approximated by binning the rows of the analyte band at a given time and then calculating the FWHM value from the resulting graph. This process is depicted in Figure 7.

The skew gives direct indication of the stretching caused by the corner. For turn designs with an arbitrary geometry, the stretching of the analyte band may not be linear as it is in Figure 2. A large skew suggests that the band will broaden more than the FWHM indicates, since diffusion will eventually force the analyte band back to a Gaussian plug.

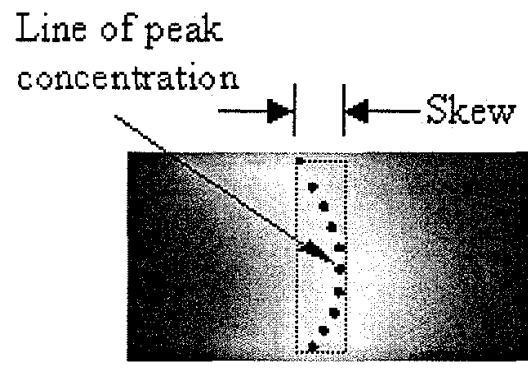

Figure 8. Determination of skew from band shape with nonlinear stretching. The axial width of the line of peak concentration is used to give an estimate of the skew. This modified definition gives the same result for analyte bands that are linearly skewed.

Simulations using MEMCAD verify the large skew predicted by Eq. 3. A sample simulation is shown in Figure 9 and compared with bleached fluorescence imaging in the same geometry. The extracted skews for the simulation and experiment shown in Figure 9 are $750 \mu \mathrm{m}$ and $800 \mu \mathrm{m}$, respectively.

Results from visualization of a compensating corner design are compared to MEMCAD simulations in Figure 10. A closer

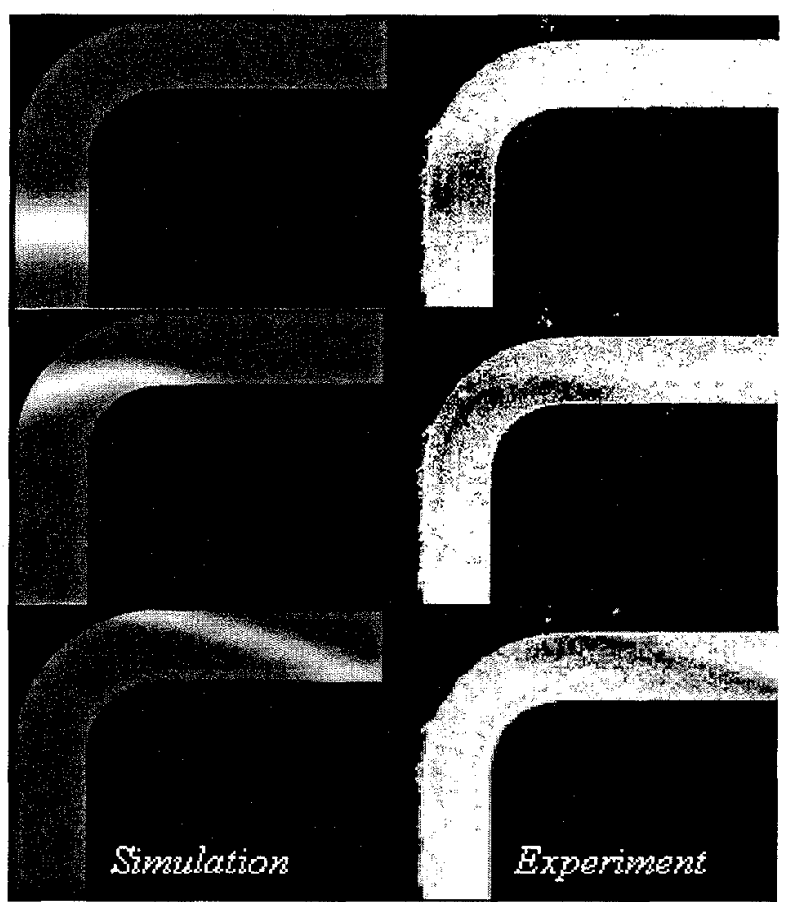

Figure 9. Simulation (left) and bleached fluorescence visualization (right) of an analyte band traveling around a constant radius corner. In both cases the channel is $250 \mathrm{\mu m}$ wide. In the experiments, the channels were approximately $40 \mu \mathrm{m}$ deep.

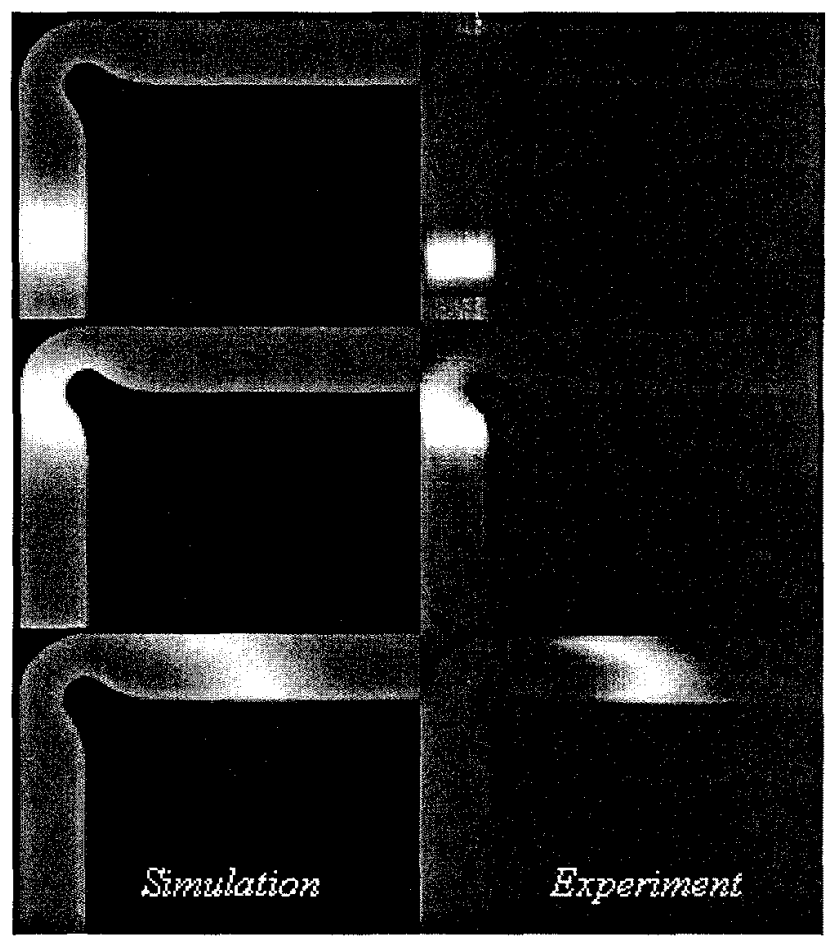

Figure 10. Simulation (left) and caged fluorescence visualization (right) of an analyte band traveling around a compensating corner design. In both cases the channel is $250 \mu \mathrm{m}$ wide. In the experiments, the channels were approximately $40 \mu \mathrm{m}$ deep. 
look at the final band shape, as seen in Figure 11, reveals that the simulation accurately reproduces details of the band shape seen in the experiment.

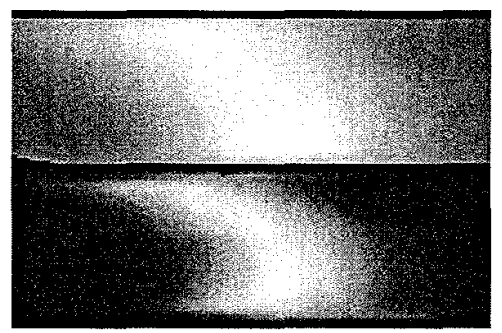

Figure 11. Close-up comparison of the band shape seen in the final frames of Figure 10. Simulation image is shown above the experimental image. Note that the parabolic shape, although more evident in the experimental images, is also apparent in the simulation. Close inspection of the trailing end of the analyte bands shows good agreement in the amount that the bottom edge of the band leads the top edge.

Table 1 summarizes the performance of four compensating corner designs and compares the skew extracted from matching simulations and experiments. Both experiments and simulations show that the compensating corners reduce the skew by as much as $75 \%$ when compared to the constant radius corners. However, Table 1 reveals some disagreement between simulation and experiment. Much of this error can be attributed to small amounts

Table 1. Comparison of skews obtained from simulations and experiments for a sample of compensating corners. The starred entries indicate measurements that were slightly perturbed by pressure driven flow. The last row gives skew extracted from the experiments and simulations shown in Figure 10.

\begin{tabular}{|c|c||c|c|}
\hline $\begin{array}{c}\text { Constriction } \\
\text { Ratio }\end{array}$ & $\begin{array}{c}\text { Recovery } \\
\text { ratio }\end{array}$ & $\begin{array}{c}\text { Skew } \\
(\exp ) \\
\mu \mathrm{m}\end{array}$ & $\begin{array}{c}\text { Skew } \\
(\mathrm{sim}) \\
\mu \mathrm{m}\end{array}$ \\
\hline 0.5 & 1 & N/A & 100 \\
\hline 0.5 & 2 & $200^{*}$ & 85 \\
\hline 0.5 & 3 & $170^{*}$ & 110 \\
\hline 0.65 & 1 & 140 & 135 \\
\hline
\end{tabular}

of pressure-driven flow, as we have found that slight imbalances in the wells (height differences of a 1-2 millimeters) can significantly disturb the skew measurements. We are currently working on new techniques to help more easily eliminate this pressure-driven flow.

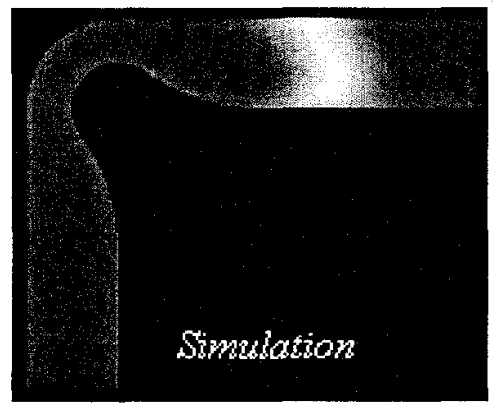

Figure 12. Simulation of a more optimized design. In this figure, only the final shape of the analyte band is shown. By adjusting the parameters for the optimized geometry, decreases in the skew and FWHM have been obtained.
Figure 12 demonstrates a design that is superior to the one shown in Figure 10. This design was the intended shape for the turn shown in Figure 10 but inaccuracies in the prototyping process resulted in a less successful design. We are currently working to improve these manufacturing methods.

We have also begun working to develop compensating corner designs for 180 degree turns, because we believe this design will provide more efficient use of space on a compact microfluidic device. Eq. 3 shows that the skew for a 180 degree turn is approximately 6 times the width of the channel. This large skew is evident in Figure 13.

Since the skew will decrease linearly with the reduction in the width of the channel, one method of minimizing skew is to taper the channel to an arbitrarily small width before the turn, and then return to the full width after the turn. Even if the tapering can be performed without increasing dispersion, there are many reasons why this technique may be problematic. The voltage drop across the turn will increase linearly with the reduction of the

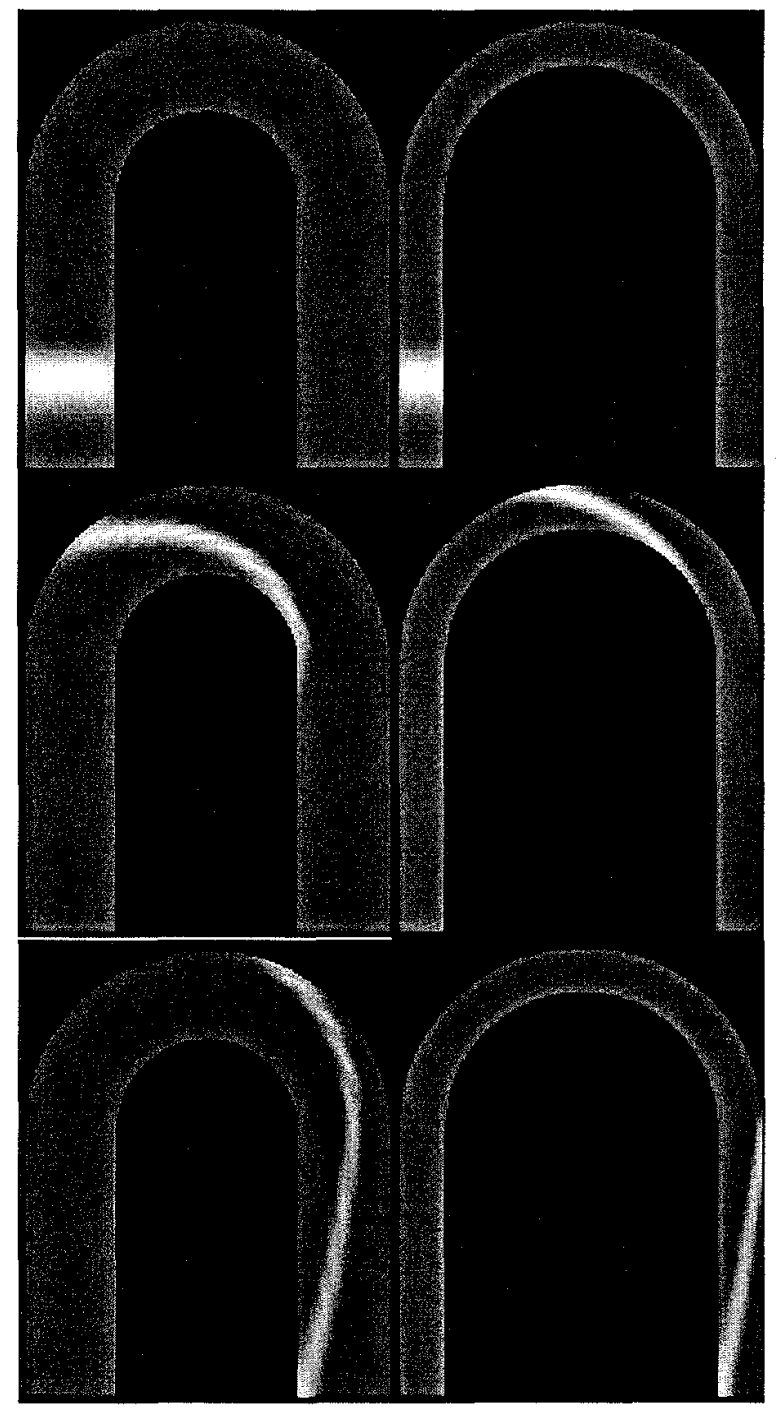

Figure 13. Simulations of an analyte band traveling through two constant radius, 180 degree turn designs. For the purpose of comparing to compensating corner designs, both $250 \mu \mathrm{m}$ and 125 $\mu \mathrm{m}$ wide designs are shown. 
channel width and therefore very narrow turns will reduce the field strength in the straight portions of the separation channel. Furthermore, the increased field strength in the turn will cause unwanted Joule heating and temperature rise, possibly leading to bubble formation in the turns.

Figure 14. presents simulations of an analyte band traveling through two different compensating corner designs. Although Figure 14 suggests that there is room for improvement, this design already reduces the skew by at least a factor of 5 as compared to the constant radius turns shown in Figure 13.

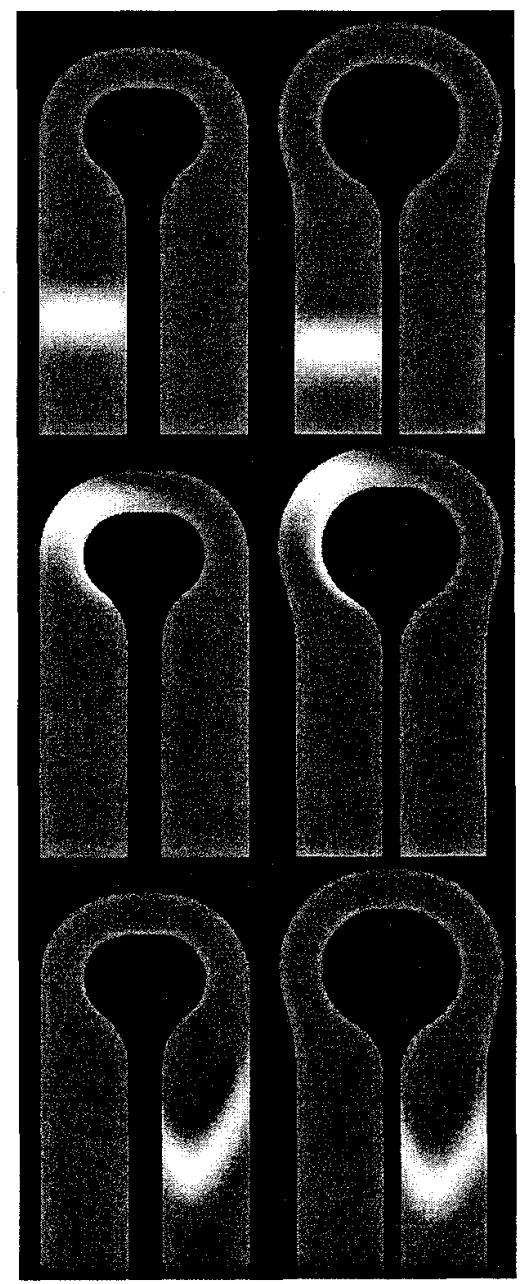

Figure 14. Simulations of two compensating, 180 degree turn designs. The first turn is essentially two of the 90 degree turns connected by a straight section. The second design incorporates an additional curvature along the outside of the turn.

Note that both of the designs shown in Figure 14 perform better than a constant radius turn having a width equal to the constriction width in the compensating designs. This enhanced performance is achieved by combining width reductions with geometry that manipulates the electric field as discussed in Figure 4. Therefore it is possible to reduce skew without the detrimental effects caused by extremely narrow constant radius turns.

\section{CONCLUSIONS}

This paper has described compensating corner designs that greatly reduce the sample dispersion caused by turns in a serpentine separation channel. Simulations were used to design the geometry of the compensating corners, and caged- and bleached fluorescence visualization techniques were used to validate the designs. The new compensating corners should be useful in realizing miniaturized electrophoresis systems that require long channels within a small area. Because of the good agreement between the simulations and experiments, we believe that simulation-based design of complete multi-turn $C E$ separation channels can be carried out, resulting in microanalytical systems with greatly improved separation efficiencies.

\section{ACKNOWLEDGEMENTS}

J. I. Molho is supported by a Hewlett-Packard Stanford Graduate Fellowship. A. E. Herr is supported by a National Science Foundation Graduate Research Fellowship. The authors would like to acknowledge the support of the Defense Advanced Research Projects Agency contract F33615-98-1-2853 with Dr. A. Lee as monitor.

\section{REFERENCES}

1. A. Manz, D. J. Harrison, E. Verpoorte, et al., "Planar Chips Technology For Miniaturization of Separation Systems : a Developing Perspective in Chemical Monitoring", Advances in Chromatography, 33 1-66 (1993).

2. C. S. Effenhauser, "Integrated chip-based microcolumn separation systems", Topics in Current Chemistry, 194 51-82 (1998).

3. C. T. Culbertson, S. C. Jacobson, and J. M. Ramsey, "Dispersion sources for compact geometries on microchips", Analytical Chemistry, 70(18) 3781-3789 (1998).

4. E. Grushka, R. M. McCormick, and J. J. Kirkland, "Effect of temperature gradients on the efficiency of capillary zone electrophoresis separations", Analytical Chemistry, 61(3) 241-246 (1989).

5. R. F. Probstein, Physicochemical hydrodynamics : an introduction. 2nd ed. 1994, New York: John Wiley \& Sons. 400.

6. E. B. Cummings, S. K. Griffiths, R. H. Nilson, et al., "Conditions for similitude between the fluid velocity and electric field in electroosmotic flow", Analytical Chemistry, (in review).

7. B. P. Mosier and J. G. Santiago. "Bleached Fluorescence Imaging", to be presented at the 2000 ASME International Mechanical Engineering Congress and Exposition, Orlando, FL.

8. P. M. St. John, M. Deshpande, J. Molho, et al. "Mctrology and Simulation of Chemical Transport in Microchannels", Solid State Sensor and Actuator Workshop, Hilton Head Island, South Carolina, Transducers Research Foundation (1998).

9. P. H. Paul, M. G. Garguilo, and D. J. Rakestraw, "Imaging of pressure- and electrokinetically driven flows through open capillarics", Analytical Chemistry, 70(13) 2459-2467 (1998). 